\title{
Real-Time Estimation of the Road Bank and Grade Angles with Unknown Input Observers
}

\author{
E. Hashemi, R. Zarringhalam, A. Khajepour, W. Melek, A. Kasaiezadeh, and S-K. Chen
}

\begin{abstract}
This paper proposes an approach for estimation of the road angles independent from the road friction conditions. The method employs unknown input observers on the roll and pitch dynamics of the vehicle. The correlation between the road angle rates and the pitch/roll rates of the vehicle are also investigated to increase the accuracy. Dynamic fault thresholds are implemented in the algorithm to ensure reliable estimation of the vehicle body and road angles. Performance of the proposed approach in reliable estimation of the road angles is experimentally demonstrated through vehicle road tests. Road test experiments include various driving scenarios on different road conditions to thoroughly validate the proposed approach.
\end{abstract}

\section{INTRODUCTION}

$\mathbf{R}$ OAD grade and bank angles considerably affect the vehicle dynamics and measured accelerations. Road angles consequently play a key role in the vehicle state estimation and stability. Several studies investigating the vehicle stability control and state estimation have been carried out based on known road angles [1]-[3]. Direct measurement of these angles in real-time is not practical for commercial vehicles due to costs. Therefore, recent developments in vehicle's active safety systems have underlined the need for real-time estimation of the road bank and grade angles as addressed by many recent studies.

Several studies focus on estimation of road inclinations while assuming the road friction condition is known. A method for dynamic estimation of the road bank angle is discussed in [4], in which the roll and lateral dynamics are used to develop the bank angle estimator. The steady-state approximation of the bank angle is used as a reference to calculate the estimation error and design the observer. This steady-state approximation is obtained using a linear vehicle model by implementing road friction information and tire characteristics. To reduce the effects of inaccuracies in transient conditions, a dynamic factor based on the understeer coefficient in high-friction scenarios is integrated with the observer. Practical problems in terms of stability control associated with estimation stability due to switching between the steady-state and transient conditions should be investigated. Zhao et al. introduced a sliding-mode observer in [5] for the velocity estimation with the road angle adaptation. Their method employs a tire model that requires the road friction and tire parameters. Menhour et al. suggest an unknown input sliding-mode observer in [6] to estimate the road bank angle. Their method employs a linear bicycle handling model for the vehicle, which needs tires' cornering stiffness and road friction information subsequently.

Alternatively, to address the road friction uncertainties, some studies identify the road friction conditions simultaneously, which may be challenging in itself because of the issues arising from lack of excitations, tire models, etc. Grip et al. suggest a nonlinear vehicle sideslip observer in [7] that incorporates time-varying gains and road friction parameters to estimate the longitudinal/lateral velocities and road angles using a tire model. Their method suggests concurrent estimation of the vehicle states, road angles, and the road condition. A time-varying observer is utilized in [8] by Grip et al. for the concurrent estimation of the road bank and the road-tire friction characteristics. They also modulate the observer gains based on a set of practical driving scenarios to improve the performance on low-friction surfaces.

Some approaches do not implement the knowledge of the road friction, but do not isolate the vehicle roll/pitch dynamics from the road inclinations. A road angle estimation is proposed by Hahn et al. in [9]. The vehicle pitch/roll induced by the suspension deflection is not separated from the road grade/bank angles. Imsland et al. suggested a nonlinear observer for the bank angle estimation in [10] to accommodate various road conditions and compared their method with an extended Kalman filter from the view point of numerical complexity. An unknown input observer is also proposed in [11] to estimate the lateral states of the vehicle as well as the bank angle. In their study, the road bank angle is assumed to be constant and its time-varying characteristics have not been taken into account in the error dynamics. A proportional integral $H_{\infty}$ filter is proposed by Kim et al. in [12]. They modified a bicycle model and made the estimation algorithm more robust against model and measurement uncertainties. In their model, the vehicle roll is not separated from the road bank.

Other literature has offered methods independent from the road friction and has included roll/pitch dynamics with additional measurements. Utilizing a tire model and steering torque measurement, Carlson et al. offer a methodology for the separation of the road angles from the induced vehicle angles in [13] to avoid vehicle rollover. Ryu et al. used two-antenna GPS receivers to estimate the road bank and compensate the corresponding roll effect on the vehicle state estimator in [14]. Roll dynamic parameters are also identified in their method. Hsu and Chen in [15] provide a modelbased estimation approach for the road angles. Their method combines multiple roll and pitch models and a switching observer scheme. However, the knowledge of the vehicle yaw angle, which is not accessible in commercial vehicles is required in their proposed observer.

To summarize, three main challenges exist in the current studies on the road angle estimation: a) unknown road friction conditions and tire parameters; b) incorporating effects of the vehicle roll and pitch angles; c) using available sensors and available measurements. Therefore, an estimation approach 
which tackles these challenges will be promising. This paper proposes a structure independent of the road friction to estimate the road angles without limiting assumptions. The roadbody kinematics is also investigated to relate the measured angle rates, vehicle body motion, and the rate of change of the road angles. The proposed estimation scheme operates in different driving scenarios as verified by road test experiments.

This article has been divided into four sections. Section II includes estimation of the vehicle body's angles, observer development on the roll/pitch dynamics, and the road-vehicle kinematics. An unknown input observer is also proposed in section II for the estimation of the road bank and grade angles. The road experiments to verify the approach in various maneuvers and driving conditions are presented in section III. Finally, conclusions are provided in section IV.

\section{ESTIMATION OF THE ROAD ANGLES}

The proposed estimation structure is depicted in Fig. 1. An unknown input observer is developed to estimate the road bank and grade angles. The Sprung mass kinematic model provides vehicle body angles $\bar{\phi}_{v}, \bar{\theta}_{v}$ for the unknown input estimator.

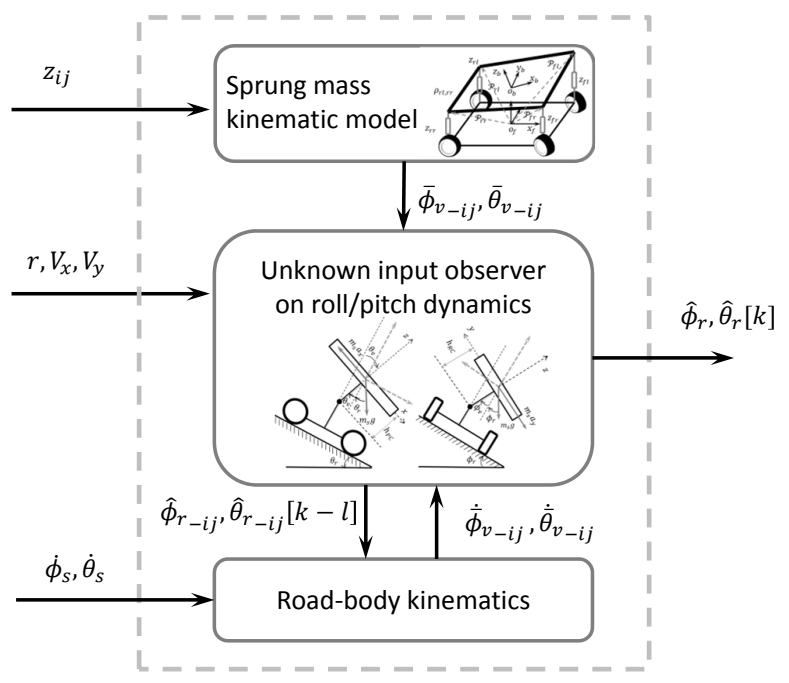

Fig. 1: The proposed structure for the road angle estimation

The body angles are estimated using corners' displacements measured by the suspension height sensors installed at corners. The Road-body kinematics module is employed to relate the vehicle's frame, body, and road angles. This module relates the road angle rates and the measured angles rates by the sensors attached to the vehicle body, and provides time derivatives $\dot{\bar{\phi}}_{v_{-i j}}, \dot{\bar{\theta}}_{v_{-i j}}$ of the vehicle body angles. The Unknown input observer module uses estimated vehicle angles and their rates for the road bank/grade estimation. Details for each block are presented in the following subsections.

\section{A. Sprung mass kinematics}

The sprung mass kinematics is used to estimate the vehicle's body roll and pitch angles $\phi_{v}, \theta_{v}$ using corners' displacements $z_{i j}$. These displacements are measured by the suspension height sensors installed at corners. A schematic of the sprung mass model and the positions of the suspension height sensors are depicted in Fig. 2

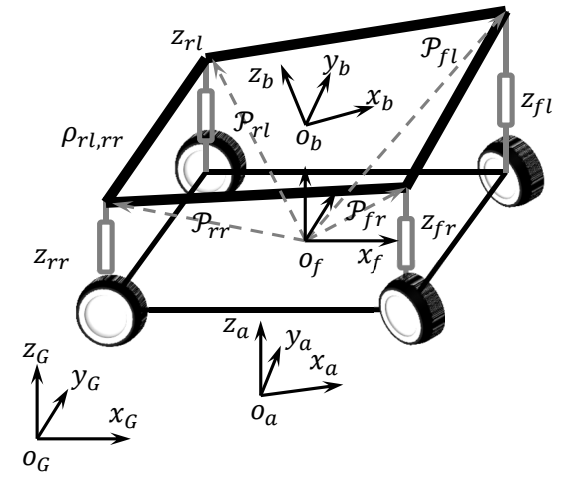

Fig. 2: Height sensors and sprung mass kinematics

The auxiliary coordinates $\left(x_{a}, y_{a}, z_{a}\right)$ is a right-handed orthogonal axis system obtained by rotating the global coordinates about the $z_{G}$ axis by the vehicle yaw angle $\psi$. The intermediate axis system $\left(x_{i}, y_{i}, z_{i}\right)$ is given by pitch rotation $\theta$ about the $y_{a}$ axis (from the auxiliary coordinates) [16]. The vehicle frame coordinates $\left(x_{f}, y_{f}, z_{f}\right)$ is also a righthanded orthogonal axis system located at the center of the frame on undeformed body. Thus, it is parallel to the plane of the road. The subscript $b$ represents the coordinates attached to the vehicle body as can be seen in Fig. 2. The sensor position vectors in the frame coordinate system $\left(x_{f}, y_{f}, z_{f}\right)$ are described with $i \in\{f, r\}$ (front and rear tracks) as follows:

$$
\begin{aligned}
& \mathcal{P}_{i L}=\left[\begin{array}{lll}
l_{i} & \operatorname{Tr}_{i} / 2 & z_{i L}
\end{array}\right]^{T} \\
& \mathcal{P}_{i R}=\left[\begin{array}{lll}
l_{i} & -T r_{r} / 2 & z_{i R}
\end{array}\right]^{T},
\end{aligned}
$$

where $l_{f}$ and $l_{r}$ are the longitudinal distances between the origin $O_{f}$ and the front and rear axles, respectively. The front and rear track widths are denoted by $T r_{f}$ and $T r_{r}$, respectively. Relative position vectors $\rho_{i j, m n}$ between two corners can be obtained by:

$$
\rho_{i j, m n}=\mathcal{P}_{m n}-\mathcal{P}_{i j},
$$

The normal vector for the sprung mass plane is then expressed as the cross product of any two relative position vectors:

$$
\mathcal{N}=\rho_{i j, m n} \times \rho_{i j, p q},
$$

in which the subscripts $i j, m n, p q \in\{f L, f R, r L, r R\}$ represent front-left $(f L)$, front-right $(f R)$, rear-left $(r L)$, and rear-right $(r R)$ corners. Therefore, using any three suspension height sensor data and corner positions, the respective normal vectors can be written as $\mathcal{N}_{-f L}=\rho_{r L, r R} \times \rho_{r R, f R}$, $\mathcal{N}_{-f R}=\rho_{f L, r L} \times \rho_{r L, r R}, \quad \mathcal{N}_{-r L}=\rho_{r R, f R} \times \rho_{f R, f L}$, and $\mathcal{N}_{-r R}=\rho_{f L, r L} \times \rho_{f L, f R}$ where the subscript $-i j$ represents a scenario in which the suspension height provided by sensor $i j$ is not used. Subsequently, components 
$\mathcal{N}_{-i j}=\left[\begin{array}{lll}\mathcal{N}_{-i j}^{x} & \mathcal{N}_{-i j}^{y} & \mathcal{N}_{-i j}^{z}\end{array}\right]^{T}$ are used to estimate the vehicle angles. The roll and pitch angles $\bar{\phi}_{v_{-i j}}, \bar{\theta}_{v_{-i j}}$ can be written with incorporation of the corresponding normal vector $\mathcal{N}_{-i j}$ as follows:

$$
\bar{\phi}_{v_{-i j}}=\cos ^{-1} \frac{\mathcal{N}_{-i j}^{y}}{\left\|\mathcal{N}_{-i j}\right\|}, \quad \bar{\theta}_{v_{-i j}}=\cos ^{-1} \frac{\mathcal{N}_{-i j}^{x}}{\left\|\mathcal{N}_{-i j}\right\|} .
$$

Four estimates for the vehicle roll angle, and four estimates for the vehicle pitch angle, can be obtained using different combinations of the suspension sensors, and a weighted average will be used to have reliable estimates in case of existing outlier data due to uneven surfaces at each corner as follows.

The four estimates for the vehicle's roll and pitch angles from (4) (four combinations of set of three corners) are examined to check the possibility of being an outlier because of road disturbances, such as bumps and uneven surfaces at each corner. Validity of the vehicle's roll/pitch angles is checked at two stages. First, all four angles $\phi_{v_{-i j}}, \bar{\theta}_{v_{-i j}}$ are compared to each other with variance checking scheme to eliminate the one with the largest deviation. Second, for each corner, the residuals of the vehicle angle rates are defined as the difference between the time derivatives of the estimated angles $\dot{\bar{\phi}}_{v_{-i j}} \dot{\bar{\theta}}_{v_{-i j}}$ at $200[H z]$ and the measured angle rates $\dot{\phi}_{s}, \dot{\theta}_{s}$ of vehicle:

$$
R_{\dot{\bar{\phi}}_{-i j}}=\left|\dot{\phi}_{s}-\dot{\bar{\phi}}_{v_{-i j}}\right|, \quad R_{\dot{\bar{\theta}}_{-i j}}=\left|\dot{\theta}_{s}-\dot{\bar{\theta}}_{v_{-i j}}\right| .
$$

When there is no disturbance at each corner, all residuals of corners $R_{\dot{\phi}_{-i j}}, R_{\bar{\theta}_{-i j}}$ fall below a certain threshold $T_{q}=T_{s q}+$ $T_{e q}\left(\left|a_{x}\right|+\left|a_{y}\right|\right)$ where $q \in\{\phi, \theta\}$. The static minimum value for the threshold is denoted by $T_{s q}$, and $T_{e q}$ introduces the effect of longitudinal/lateral excitations to the threshold. Lowpass filters can also be utilized to smooth the time derivatives of the estimated angles. After isolation of the outliers by the mentioned two tests, weighted vehicle angles $\bar{\phi}_{v_{-i j}}, \bar{\theta}_{v_{-i j}}$ from each combination of the three corner sensors are employed in the estimation of the vehicle's roll/pitch angles as follows:

$$
\bar{\phi}_{v}=\sum_{i j} \gamma_{-i j} \bar{\phi}_{v_{-i j}}, \quad \bar{\theta}_{v}=\sum_{i j} \gamma_{-i j} \bar{\theta}_{v_{-i j}}
$$

where the weight of each three sensor combination is denoted by $\gamma_{-i j}$ and is set to 0.25 (average of the calculated angles) for the case in which there is no outlier. Whenever a disturbance or an outlier is detected in the suspension height sensor measurement at a corner, three weights will be zero since the subsequent three estimated body angles by such an outlier are not reliable. For instance, when there is a disturbance at the front-right suspension height sensor, its residuals exceed the thresholds $T_{\phi}, T_{\theta}$, thus the only non-zero weight will be $\gamma_{-f R}$ and all other three weights will be zero. When more than one outlier is identified, the estimated vehicle roll/pitch angles are not valid and the algorithm incorporates the previously estimated valid body angles. The estimated vehicle angles (6) are employed for the unknown input observer to estimate the road angles as will be discussed in the following subsection.

\section{B. Unknown input observer for road angle estimation}

This section presents a methodology to estimate the road angles using unknown input observers (UIO). The problem of constructing an observer for systems with unknown inputs (epitomizing disturbances, faults, and uncertainties) has been widely tackled in the literature with realizing full and reducedorder observers [17]-[20] and turns out to be considerably useful in diagnosing system faults [21]-[23]. A general form of the UIO is utilized in this section to estimate the unknowns (terms representing the road angles) with implementation of the vehicle body angles and their rates as the outputs. Roll and pitch dynamic models are used for the proposed UIO and graphically illustrated in Fig. 3. The road bank and grade angles are denoted by $\phi_{r}$ and $\theta_{r}$ respectively.

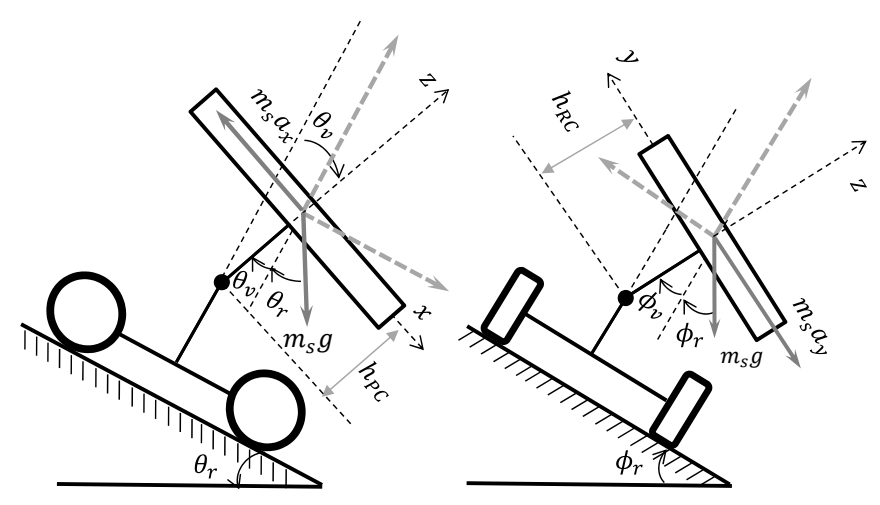

Fig. 3: Roll and pitch models with the road angles

Employing vehicle kinematics, the roll and pitch dynamics can be expressed as $\dot{x}_{\phi}=A_{\phi} x_{\phi}+B_{\phi} u_{\phi}$ and $\dot{x}_{\theta}=A_{\theta} x_{\theta}+$ $B_{\theta} u_{\theta}$ where the states are $x_{\phi}=\left[\begin{array}{ll}\phi_{v} & \dot{\phi}_{v}\end{array}\right]^{T}, x_{\theta}=\left[\begin{array}{ll}\theta_{v} & \dot{\theta}_{v}\end{array}\right]^{T}$ [24], and the roll and pitch angles of the sprung mass are denoted by $\phi_{v}, \theta_{v}$. The roll and pitch dynamics yield the following:

$$
\begin{aligned}
& \dot{x}_{\phi}=\left[\begin{array}{cc}
0 & 1 \\
\frac{-K_{\phi}}{I_{x}+m_{s} h_{R C}^{2}} & \frac{-C_{\phi}}{I_{x}+m_{s} h_{R C}^{2}}
\end{array}\right] x_{\phi}+\left[\begin{array}{c}
0 \\
\frac{m_{s} h_{R C}}{I_{x}+m_{s} h_{R C}^{2}}
\end{array}\right] u_{\phi}, \\
& \dot{x}_{\theta}=\left[\begin{array}{cc}
0 & 1 \\
\frac{-K_{\theta}}{I_{y}+m_{s} h_{P C}^{2}} & \frac{-C_{\theta}}{I_{y}+m_{s} h_{P C}^{2}}
\end{array}\right] x_{\theta}+\left[\begin{array}{c}
0 \\
\frac{m_{s} h_{P C}}{I_{y}+m_{s} h_{P C}^{2}}
\end{array}\right] u_{\theta},
\end{aligned}
$$

in which road bank and grade angles $\phi_{r}, \theta_{r}$ appear in unknown inputs $u_{\phi}, u_{\theta}$. In (7) and (8), the distances between the roll/pitch axes and the center of gravity are denoted by $h_{R C}$ and $h_{P C}$. The moments of inertia about the roll and pitch axes parallel to the frame coordinate system are shown by $I_{x}, I_{y}$. Roll/pitch stiffness $K_{\phi}, K_{\theta}$ and damping $C_{\phi}, C_{\theta}$ are used for derivation of the roll and pitch dynamics. The unknown longitudinal and lateral inputs are denoted by:

$$
\begin{gathered}
u_{\phi}=\dot{V}_{y}+r V_{x}+g \sin \left(\bar{\phi}_{v}+\phi_{r}\right), \\
u_{\theta}=-\dot{V}_{x}+r V_{y}+g \sin \left(\bar{\theta}_{v}+\theta_{r}\right),
\end{gathered}
$$

in which $\phi_{r}$ and $\theta_{r}$ show the road bank and grade respectively. The vehicle's yaw rate $r$ is measured by the available stock 
inertial measurement unit (IMU) sensor. The longitudinal and lateral velocities $V_{x}, V_{y}$ can be measured by a GPS or can be estimated using linear, nonlinear, or Kalman-based observers provided in the literature [1], [3], [25]-[28]

Therefore, systems (7), (8) can be rewritten as $\dot{x}_{q}=$ $A_{q} x_{q}+B_{q} u_{q}$ and $y_{q}=C_{q} x_{q}+D_{q} u_{q}$ with state vector $x \in \mathbb{R}^{2}$, unknown input vector $u_{q} \in \mathbb{R}$, output $y \in \mathbb{R}^{2}$, and system matrices $A_{q}, B_{q}, C_{q}, D_{q}$ of appropriate dimensions where $\left[\begin{array}{ll}B_{q} & D_{q}\end{array}\right]^{T}$ is full-column rank and $q \in\{\phi, \theta\}$. The road angles also appear as unknown parameters in roll/pitch dynamics (7) and (8). An unknown input observer [19], [22] is designed to estimate the road bank $\phi_{r}$ and road grade $\theta_{r}$ (unknown inputs $u_{q}$ ) using vehicle body's roll/pitch angles $\bar{\phi}_{v}, \bar{\theta}_{v}$ and their rates $\dot{\bar{\phi}}_{v}, \dot{\bar{\theta}}_{v}$ as measurements. Derivation of the vehicle roll/pitch rates is discussed at the end of the next subsection Road-body kinematics.

To develop the observer for practical application, discretization of the systems (7), (8) is performed by the step-invariance method [29], because of its precision and response characteristics. Input to the continuous-time systems (7) and (8) is the hold signal $u_{q}[k]=u_{q}(t[k])$ for a period (sample time $T_{s}$ ). Then, the discrete-time system has the state, input, and output matrices $\bar{A}_{q}=e^{A_{q}(t) T_{s}}, \bar{B}_{q}=\int_{0}^{T_{s}} e^{A_{q}(t) \tau} B_{q}(t) d \tau, \bar{C}_{q}=$ $C_{q}, \bar{D}_{q}=D_{q}$. Thus, the discrete-time form of the roll and pitch dynamics yields the foloowing:

$$
\begin{aligned}
x_{q}[k+1] & =\bar{A}_{q} x_{q}[k]+\bar{B}_{q} u_{q}[k] \\
y_{q}[k] & =\bar{C}_{q} x_{q}[k]+\bar{D}_{q} u_{q}[k],
\end{aligned}
$$

The system (11) has an $L$-delay inverse if it is feasible to uniquely recover the unknown input $u_{q}[k]$ from the initial state $x[0]$ and outputs up to time step $k+L$ for a positive integer $L$; the least integer $L$ which leads to $L$-delay inverse is the inherent delay of the system. The upper bound on the inherent delay is defined as $L \triangleq n-\operatorname{Null}\left(\bar{D}_{q}\right)+1$ in [30]. The output equation from (11) can be accumulated for $L$ time steps:

$$
y_{q}[0: L]=\mathcal{O}_{L q} x[0]+\mathcal{J}_{L q} u_{q}[0: L] .
$$

where $\mathcal{J}_{L q}$ is the invertibility matrix of the system (11), $L$ is required for recovery of $x_{q}[0]$ from the output $y_{q}[0: L]$, and $\mathcal{O}_{L q}$ is the observability matrix for the pair $\bar{A}_{q}, \bar{C}_{q}$. Observability and invertibility matrices are provided in the appendix. When the start point is the sample time $k,(12)$ yields $y_{q}[k: k+L]=\mathcal{O}_{L q} x[k]+\mathcal{J}_{L q} u_{q}[k: k+L]$.

The unknown input observer for a positive arbitrary $L$ results in the following estimator, which provides the states $\hat{x}_{\phi}[k], \hat{x}_{\theta}[k]$ as well as unknown inputs $\hat{u}_{\phi}[k], \hat{u}_{\theta}[k]$ :

$$
\begin{aligned}
& \hat{x}_{q}[k+1]=E_{q} \hat{x}_{q}[k]+F_{q} y_{q}[k: k+L], \\
& \hat{u}_{q}[k]=\left[\begin{array}{c}
\bar{B}_{q} \\
\bar{D}_{q}
\end{array}\right]^{-1}\left[\begin{array}{c}
\hat{x}_{q}[k+1]-\bar{A}_{q} \hat{x}_{q}[k] \\
y_{q}[k]-\bar{C}_{q} \hat{x}_{q}[k]
\end{array}\right],
\end{aligned}
$$

where $E_{q}$ and $F_{q}$ are observer gain matrices obtained by pole placement as will be described in the following. The general form of the discrete-time system (11) with state vector $x_{q} \in \mathbb{R}^{n}$, output $y_{q} \in \mathbb{R}^{m}$, and unknown input vector $u_{q} \in \mathbb{R}^{p}$ has the observability and invertibility matrices
$\mathcal{O}_{L q} \in \mathbb{R}^{m(L+1) \times n}, \mathcal{J}_{L q} \in \mathbb{R}^{m(L+1) \times p(L+1)}$ and observer gain matrices $E_{q} \in \mathbb{R}^{n \times n}, F_{q} \in \mathbb{R}^{n \times m(L+1)}$ respectively. Thereby, for the discretized form of the systems (7), (8), the observability matrix, invertibility matrix, and observer gain matrices are $\mathcal{O}_{L q} \in \mathbb{R}^{2(L+1) \times 2}, \mathcal{J}_{L q} \in \mathbb{R}^{2(L+1) \times(L+1)}$ and $E_{q} \in \mathbb{R}^{2 \times 2}, F_{q} \in \mathbb{R}^{2 \times 2(L+1)}$ when the vehicle body's roll/pitch angles and their rates $\dot{\phi}_{v}, \dot{\theta}_{v}$ are utilized as measurements.

The discrete-time estimation error for the pitch and roll dynamics can be expressed as follows using (11) and (12), and the unknown input observer (13):

$$
\begin{aligned}
e_{q}[k+1]= & \hat{x}_{q}[k+1]-x_{q}[k+1] \\
= & E_{q} x_{q}[k]+F_{q} y_{q}[k: k+L] \\
& +\left(E_{q}-\bar{A}_{q}\right) x_{q}[k]-\bar{B}_{q} u_{q}[k] \\
= & E_{q} e_{q}[k]+F_{q} \mathcal{J}_{L q} u_{q}[k: k+L] \\
& +\left(E_{q}-\bar{A}_{q}+F_{q} \mathcal{O}_{L q}\right) x_{q}[k]-\bar{B}_{q} u_{q}[k]
\end{aligned}
$$

where the smallest $L_{q}$ with upper bound $L_{q}<n-$ $\operatorname{Null}\left(\bar{D}_{q}\right)+1$ should be determined such that $\operatorname{rank}\left(\mathcal{J}_{L q+1}\right)-$ $\operatorname{rank}\left(\mathcal{J}_{L q}\right)=p$. In order to have asymptotic stability on the error dynamics (15) regardless of $x_{q}[k]$ and inputs, $E_{q}$ should be stable, i.e. $\left|\lambda_{i}\left(E_{q}\right)\right|<1, \forall i \in\{1, . . n\}$, and $F_{q}$ should simultaneously satisfy the following [22]:

$$
\begin{gathered}
F_{q} \mathcal{J}_{L q}=\left[\begin{array}{ll}
\bar{B}_{q} & 0 \ldots 0
\end{array}\right], \\
F_{q} \mathcal{O}_{L q}+E_{q}-\bar{A}_{q}=0 .
\end{gathered}
$$

The matrix $F_{q}$ is obtained from $F_{q}=M_{q} V$ where $V=$ $\left[\begin{array}{lll}0 & 0 ; I_{p} & 0\end{array}\right]$ and $M_{q}=\left[\begin{array}{ll}\bar{M}_{q} & \bar{B}_{q}\end{array}\right]$. The matrix $\bar{M}_{q}$ is chosen by a pole placement such that matrix $E_{q}=\bar{A}_{q}-\bar{B}_{q} \breve{W}_{q}-$ $\bar{M}_{q} \bar{W}_{q}$ is stable. The matrix $W_{q}=\left[\begin{array}{ll}\bar{W}_{q} & \breve{W}_{q}\end{array}\right]^{T}$ is defined as $W_{q} \triangleq V \mathcal{O}_{L q}$ in which $\breve{W}_{q}$ has $p$ rows.

The stability of the state estimation error dynamics (15), system equations (11) and the estimated unknown input (14) guarantees that $\hat{u}_{q}[k] \rightarrow u_{q}[k]$ as $k \rightarrow \infty$

Remark 1: An unknown input observer with delay $L_{q}$ can be designed for the system (11) if and only if the system is strongly detectable [19]. This is equivalent to the following conditions:

$$
\begin{gathered}
\operatorname{rank}\left(J_{L q}\right)-\operatorname{rank}\left(J_{L q-1}\right)=p \\
\operatorname{rank}\left(\left[\begin{array}{cc}
A_{q}-z I_{n} & B_{q} \\
C_{q} & D_{q}
\end{array}\right]\right)=p+n \quad \forall z \in \mathbb{C},|z| \geq 1 .
\end{gathered}
$$

Remark 2: The systems (7) and (8) with the discretized form (11) and two measurements (roll/pitch and their rates) is strongly detectable. Thus, an UIO can be designed for this system.

The road bank angle $\hat{\phi}_{r}$ is obtained employing the estimated unknown input $\hat{u}_{\phi}$ from (14), the roll input definition (9) and the vehicle's roll angle from (6) as follows:

$$
\hat{\phi}_{r}[k]=\sin ^{-1} \frac{\hat{u}_{\phi}[k]-\dot{V}_{y}[k]-r[k] V_{x}[k]}{g}-\bar{\phi}_{v}[k] .
$$


Similarly, the unknown input observer (14) is employed for the estimation of the road grade $\hat{\theta}_{r}$, which appears as an unknown input to the pitch dynamics (8). Given the vehicle's pitch angle from (6), the pitch input definition (10) and the estimated unknown input $\hat{u}_{\theta}$ from (14), the road grade is estimated as:

$$
\hat{\theta_{r}}[k]=\sin ^{-1} \frac{\hat{u}_{\theta}[k]+\dot{V}_{x}[k]-r[k] V_{y}[k]}{g}-\bar{\theta}_{v}[k] .
$$

The two measurements: roll/pitch angles from the suspension height sensors and their rates are used for the road grade and bank angle estimation employing the unknown input observer (14) and equations (20) and (21). To calculate the roll/pitch angle rates, taking time derivatives of the vehicle angles (6) is not a proper choice since it generates oscillations due to measurement noises. Filtering such noises usually imposes undesirable delays. Thus, implementing available measurements (roll/pitch rates) from the IMU seems more promising. In order to use the measured roll/pitch rates from the sensor attached to the sprung mass, transformation between the vehicle's frame coordinate and the body coordinate should be investigated. The following section focuses on the roadvehicle kinematics in order to relate the measured angle rates, vehicle body motion, and the rate of change of the road bank and grade angles.

\section{Road-body kinematics}

Euler angles $\psi, \theta, \phi$ are utilized in this section to transform from the global coordinates $\left(x_{G}, y_{G}, z_{G}\right)$ to the vehicle frame axis system shown in Fig. 2. These angles are successive rotations about $z_{G}, y_{a}$ and $x_{f}$, respectively. Using the rotation matrices, the angular velocity of the frame relative to the global axis system can be described by $\dot{\Gamma}_{f}=R_{f}^{G} \dot{\Gamma}$ where $\dot{\Gamma}_{f}=\left[\begin{array}{lll}\dot{\phi}_{f} & \dot{\theta}_{f} & \dot{\psi}_{f}\end{array}\right]^{T}$ is the rotation rate of the frame relative to the global coordinates defined in the vehicle frame-fixed coordinates, and $\dot{\Gamma}=\left[\begin{array}{lll}\dot{\phi} & \dot{\theta} & \dot{\psi}\end{array}\right]^{T}$ represents the rate of Euler angles. Defining $\dot{\Phi}=[\dot{\phi}, 0,0]^{T}, \dot{\Theta}=[0, \dot{\theta}, 0]^{T}$, and $\dot{\Psi}=[0,0, \dot{\psi}]^{T}$, one can write the rotation matrix $R_{f}^{G}$ as

$$
R_{f}^{G}=R_{x_{f}, \phi} \dot{\Phi}+R_{x_{f}, \phi} R_{y_{a}, \theta} \dot{\Theta}+R_{x_{f}, \phi} R_{y_{a}, \theta} R_{z_{G}, \psi} \dot{\Psi}
$$

in which $R_{x_{f}, \phi}$ shows the third rotation by an angle $\phi$ about the $x_{f}$ axis, $R_{y_{a}, \theta}$ is the second rotation by an angle $\theta$ about the $y_{a}$ axis, and $R_{z_{G}, \psi}$ represents the first rotation by an angle $\psi$ about the $z_{G}$ axis. Substituting rotation matrices in (22) yields the following:

$$
R_{f}^{G}=\left[\begin{array}{ccc}
1 & 0 & -S \theta \\
0 & C \phi & S \phi C \theta \\
0 & -S \phi & C \phi C \theta
\end{array}\right]
$$

in which $C *=\cos (*)$ and $S *=\sin (*)$. Road angles are defined between the vehicle frame and the auxiliary axis system $\left(x_{a}, y_{a}, z_{a}\right)$ [16]. Therefore, the angular velocity of the vehicle frame relative to the auxiliary coordinates represents the rate of change of the road angles $\dot{\Gamma}_{r}=\left[\begin{array}{lll}\dot{\phi}_{r} & \dot{\theta}_{r} & \dot{\psi}_{r}\end{array}\right]^{T}$. Transformation $\left(R_{y_{a}, \theta}\right)^{T}$ from the intermediate coordinates $\left(x_{i}, y_{i}, z_{i}\right)$ to the auxiliary one is used to relate the road and Euler angle rates as follows:

$$
\dot{\Gamma}_{r}=\left(R_{y_{a}, \theta}\right)^{T} \dot{\Phi}+\dot{\Theta}=\left[\begin{array}{ccc}
C \theta & 0 & 0 \\
0 & 1 & 0 \\
-S \theta & 0 & 0
\end{array}\right] \dot{\Gamma}
$$

Substituting $\dot{\Gamma}=\left(R_{f}^{G}\right)^{-1} \dot{\Gamma}_{f}$ into (24) results in:

$$
\begin{aligned}
\dot{\Gamma}_{r} & =\left[\begin{array}{ccc}
C \theta & S \phi S \theta & C \phi S \theta \\
0 & C \phi & -S \phi \\
-S \theta & -S \phi S \theta \tan \theta & -C \phi S \theta \tan \theta
\end{array}\right] \dot{\Gamma}_{f} \\
& =R_{r}^{f} \dot{\Gamma}_{f},
\end{aligned}
$$

in which the rotation matrix $R_{r}^{f}$ represents the transformation between the road and frame angles. The third component $\dot{\psi}_{r}$ can be neglected since the yaw rate of the road is not the concern for this study. Therefore, (25) is reduced to:

$$
\dot{\Gamma}_{r}=\left[\begin{array}{ccc}
C \theta & S \phi S \theta & C \phi S \theta \\
0 & C \phi & -S \phi
\end{array}\right] \dot{\Gamma}_{f}=\chi_{r}^{f} \dot{\Gamma}_{f},
$$

where $\dot{\Gamma}_{r}=\left[\begin{array}{ll}\dot{\phi}_{r} & \dot{\theta}_{r}\end{array}\right]^{T}$ shows the rate of the change of the road grade and bank angles. Afterwards, employing the pseudo inverse $\left(\chi_{r}^{f}\right)^{-1}$, one can express the frame rotation rates as $\dot{\Gamma}_{f}=\left(\chi_{r}^{f}\right)^{-1} \dot{\Gamma}_{r}$ from (26). The pitch and roll rate sensors are mounted on the body sprung mass which has an orthogonal axis system $\left(x_{b}, y_{b}, z_{b}\right)$. This body-fixed coordinate system is obtained by consecutive rotations of $\phi_{v}, \theta_{v}$ around the $x_{f}$ and $y_{f}$ axes of the vehicle frame coordinates, respectively. The measured rotation rate signal, $\dot{\Gamma}_{s}=\left[\begin{array}{lll}\dot{\phi}_{s} & \dot{\theta}_{s} & \dot{\psi}_{s}\end{array}\right]^{T}$ is affected by the rotation rates of the body-fixed coordinate $\dot{\Gamma}_{v}=\left[\begin{array}{lll}\dot{\phi}_{v} & \dot{\theta}_{v} & \dot{\psi}_{v}\end{array}\right]^{T}$, and the frame rotation rate as $\dot{\Gamma}_{s}=$ $\dot{\Gamma}_{v}+R_{b}^{f} \dot{\Gamma}_{f}$. Rotation matrix $R_{b}^{f}$ is from the frame-fixed axes to the body-fixed axes and is a function of the vehicle roll/pitch angles $\phi_{v}, \theta_{v}$ about the frame-fixed $x$-axis:

$$
R_{b}^{f}=\left[\begin{array}{ccc}
C \theta_{v} & S \phi_{v} S \theta_{v} & -C \phi_{v} S \theta_{v} \\
0 & C \phi_{v} & S \phi_{v} \\
S \theta_{v} & -C \theta_{v} S \phi_{v} & C \phi_{v} C \theta_{v}
\end{array}\right]
$$

The relationship between the pitch/roll rate sensor measurements, vehicle pitch/roll rate, and road angle rates can be described using (26) as

$$
\dot{\Gamma}_{s}=\dot{\Gamma}_{v}+R_{b r} \dot{\Gamma}_{r}
$$

where the rotation between the road and the body-fixed axes is denoted by the rotation matrix $R_{b r}=R_{b}^{f}\left(\chi_{r}^{f}\right)^{-1}$. An implication of (28) is that the road angle rates should be taken into account for the estimation of the vehicle angle rates $\dot{\Gamma}_{v}$.

Conclusively, replacing $\phi_{v}, \theta_{v}$ with the calculated vehicle roll/pitch angles $\bar{\phi}_{v}, \bar{\theta}_{v}$ from (6), one can summarize the relation between the estimated vehicle angle rates $\dot{\hat{\Gamma}}_{v_{-i j}}$, estimated road angle rates $\dot{\hat{\Gamma}}_{r_{-i j}}$, and the sensor measurement $\dot{\hat{\Gamma}}_{s_{-i j}}$, in a scenario without using the suspension height sensor $i j$ as

$$
\begin{aligned}
& \dot{\bar{\phi}}_{v_{-i j}}=\dot{\phi}_{s}-R_{1}\left(\bar{\phi}_{v_{-i j}}, \bar{\theta}_{v_{-i j}}\right) \dot{\hat{\phi}}_{r_{-i j}} \\
& \dot{\bar{\theta}}_{v_{-i j}}=\dot{\theta}_{s}-R_{2}\left(\bar{\phi}_{v_{-i j}}, \bar{\theta}_{v_{-i j}}\right) \dot{\hat{\theta}}_{r_{-i j}}
\end{aligned}
$$


where $R_{1}, R_{2}$ are components of $R_{b r}=\left[\begin{array}{ll}R_{1} & R_{2}\end{array}\right]^{T}$. The estimation on the roads with the combined bank and grade angles can be achieved with (29) which presents the relation between the frame, body, and road angles. Equation (29) implies that the time derivatives of the vehicle angle rates $\dot{\bar{\phi}}_{v_{-i j}}, \dot{\bar{\theta}}_{v_{-i j}}$ can be calculated with the measured vehicle angle rates $\dot{\phi}_{s}, \dot{\theta}_{s}$ and the rate of change of the road angles. Assuming the road angles change smoothly, the road angle rates are obtained by the time derivative of the estimated ones $\bar{\phi}_{r_{-i j}}[k-l], \bar{\theta}_{r_{-i j}}[k-l]$ over $l$ previous time steps. This is shown in Fig. 1, in which the estimated road angles over $l$ previous time steps and measured body's angle rates are utilized in the Road-body kinematics to estimate the vehicle angle rates. Substituting the rates (29) and allocating the weights $\gamma_{-i j}$, the roll/pitch rates of the vehicle are expressed as follows:

$$
\dot{\bar{\phi}}_{v}=\sum_{i j} \gamma_{-i j} \dot{\bar{\phi}}_{v_{-i j}}, \quad \dot{\bar{\theta}}_{v}=\sum_{i j} \gamma_{-i j} \dot{\bar{\theta}}_{v_{-i j}} .
$$

The average weight $\left(\gamma_{-i j}=0.25\right)$ is used when there is no outlier. Whenever, a disturbance exists at the corner $m n$, the residuals (5) exceed the thresholds $T_{q}$ and an outlier is detected. Therefore, three weights related to that corner are set to zero and the only non-zero weight will be $\gamma_{-m n}$. Consequently, the vehicle roll/pitch rates (30) are utilized as measurements for the unknown input observers (13) and (14) described in the previous subsection. The next section includes road tests to validate the proposed UIO (14) with measurements (6) and (30) on different roads with separate or combined bank/grade angles.

\section{RESULTS AND DISCUSSION}

Several experiments have been carried out on a four-wheelindependent-drive sport utility vehicle with specifications listed in Table I to verify the proposed estimation scheme.

TABLE I: Vehicle Parameters for Experiments

\begin{tabular}{cccc}
\hline Parameter & Unit & Value & Description \\
\hline$m, m_{s}$ & {$[\mathrm{~kg}]$} & 2260,1989 & Total \& sprung mass \\
$I_{x}, I_{y}$ & {$\left[\mathrm{~kg} . \mathrm{m}^{2}\right]$} & 967,2710 & Moments of inertia \\
$L_{f}, L_{r}$ & {$[\mathrm{~m}]$} & $1.31,1.50$ & Front/rear axles to CG \\
$R_{e}$ & {$[\mathrm{~m}]$} & 0.37 & Effective radius \\
$h_{R C}, h_{P C}$ & {$[\mathrm{~m}]$} & $0.55,0.55$ & Roll/pitch axis height \\
$K_{\phi}, K_{\theta}$ & {$[\mathrm{N} / \mathrm{m}]$} & $(1.51,2.08) \times 10^{5}$ & Roll/pitch stiffness \\
$C_{\phi}, C_{\theta}$ & {$[N . s / m]$} & $(0.63,2.52) \times 10^{4}$ & Roll/pitch damping \\
$\operatorname{Tr}_{f}, T r_{r}$ & {$[\mathrm{~m}]$} & $1.61,1.60$ & Front/rear track width \\
\hline
\end{tabular}

The vehicle's roll, pitch, and yaw rates as well as the longitudinal and lateral acceleration are measured with a 6-axis IMU (and GPS) system RT2000. The Road Angle Estimator module requires longitudinal and lateral velocities, which can be measured using the GPS or estimated by the Velocity Estimator module as shown in Fig. 4. Four suspension height measurement sensors (from Delphi Co.) are installed at four wheel positions to measure vertical displacements of each corner and estimate vehicle body's angles. Measured signals are communicated using a CAN-bus. Real-time acquisition and processing of sensory information and the developed algo-

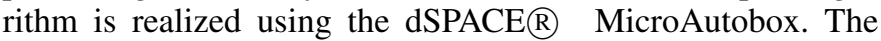

dSPACE compiles measurements for MATLAB/SIMULINK, and the controller provides control signals for the dSPACE as well. Visualization of the experiment results is performed through the ControlDesk and MATLAB/SIMULINK. The sampling frequency for the experiment is set to be $200[\mathrm{~Hz}]$.

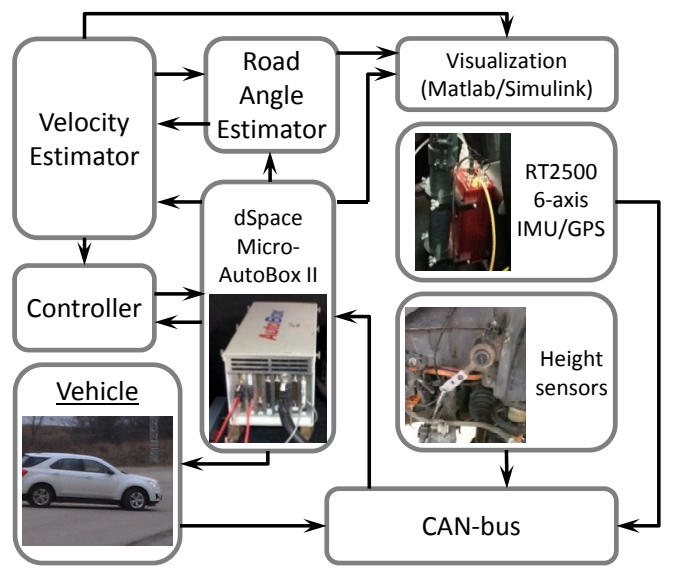

Fig. 4: The I/O and hardware layout

The performance of the estimator is experimentally examined in three cases on roads with different bank and grade conditions. The UIO gain matrices $E_{q}, F_{q}$ are provided in the appendix; the inherent delay for both roll and pitch dynamics in the observer is $L_{q}=1$, the static thresholds $T_{s \phi}=0.02, T_{s \theta}=$ 0.04 and excitation thresholds $T_{e \phi}=0.0015, T_{e \theta}=0.0019$ are also used for the road experiments.

Case 1: Acceleration/brake on the graded road

The real-time performance the estimator in a maneuver with minor steering and successive acceleration and brake on a graded road is investigated in this section. Longitudinal and lateral accelerations for this maneuver are depicted in Fig. 5 which shows excitations in the longitudinal direction.

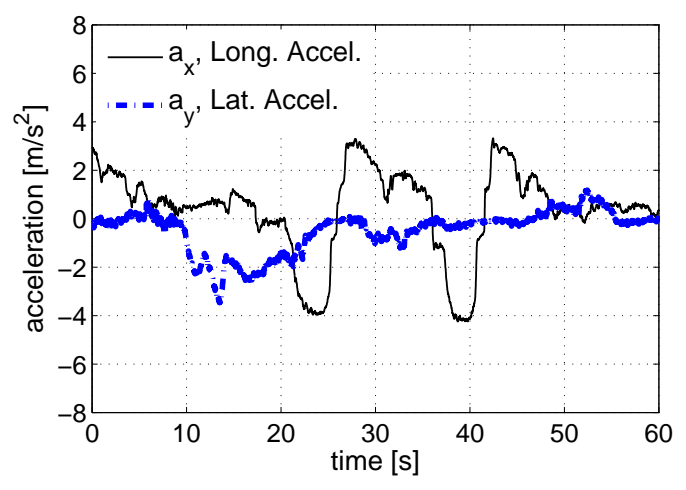

Fig. 5: Acceleration measurement on the graded road

Suspension height sensor measurements are shown in Fig. 6 that confirms several body pitch excitations due to the successive acceleration and brake. 


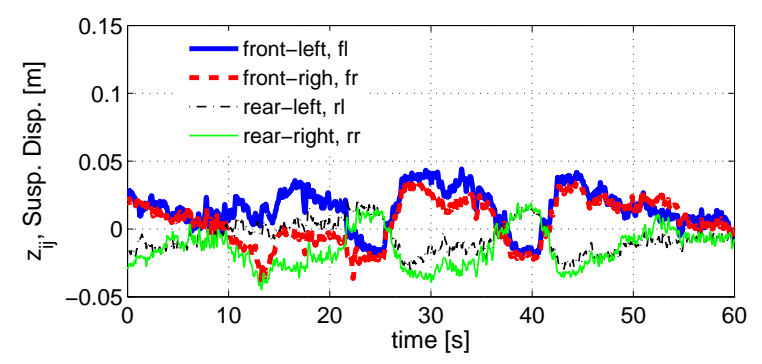

Fig. 6: Suspension height measurements on the graded road

Estimated vehicle body angles $\bar{\phi}_{v}, \bar{\theta}_{v}$ are illustrated in Fig. 7-a. There is no disturbance/outlier and the averaging method is used by the algorithm for the vehicle pitch/roll angle estimation. The estimated road grade in the ISO coordinate system is shown in Fig. 7-b, which exhibits correspondence with the measured actual grade in spite of harsh excitations on the vehicle body angles observed in Fig. 7-a.
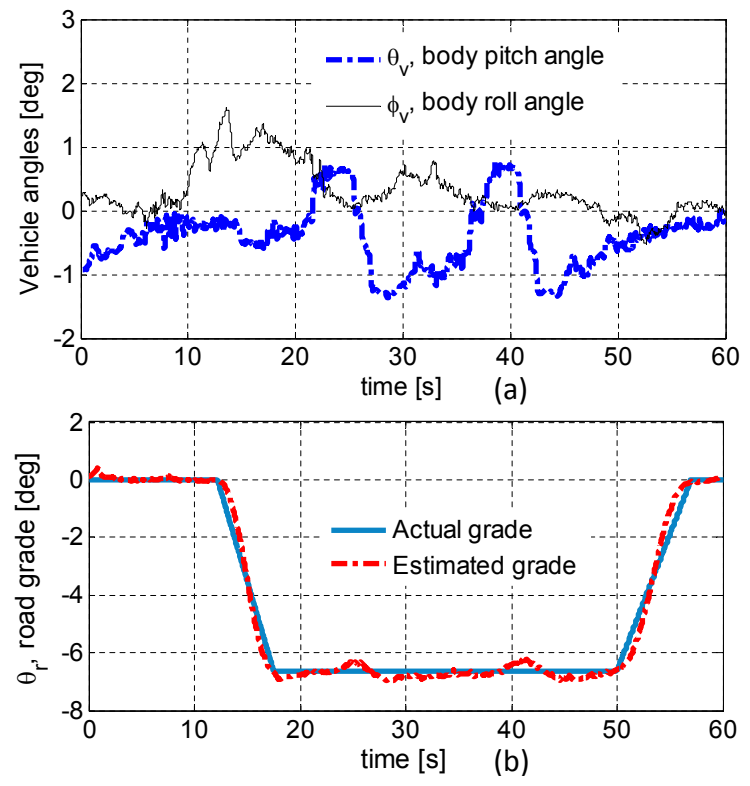

Fig. 7: Estimation results for Case1: (a) vehicle angles and (b) road grade

This substantiates that the suggested unknown input observer can accurately estimate the road grade regardless of the longitudinal (body pitch) excitations.

Case 2: Normal driving on a banked road

To distinguish between the estimated bank and grade on different roads and check the performance of the suggested estimator, a normal driving scenario with steering and lateral excitation is performed on a banked road. Figure 8 illustrates the longitudinal and lateral accelerations for this maneuver.

Variations in the lateral acceleration after $t=50[s]$ are caused by the road bank angle and the lateral excitation. Such a coupling makes the task of accurate real-time bank estimation

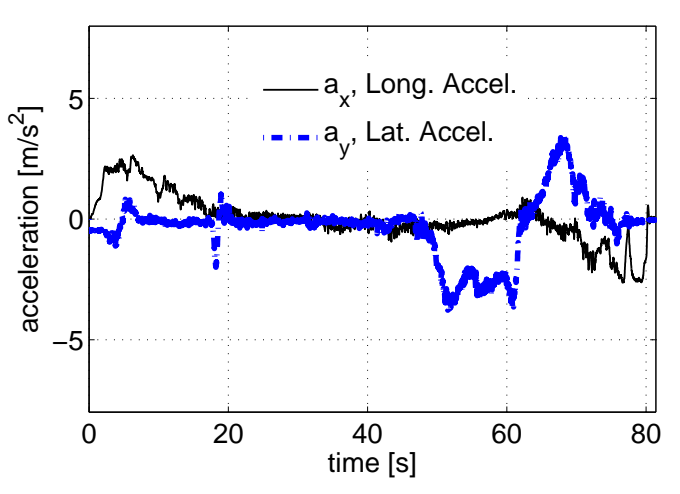

Fig. 8: Acceleration measurements on the banked road

more challenging. The displacements of each corner for this maneuver are illustrated in Fig. 9.

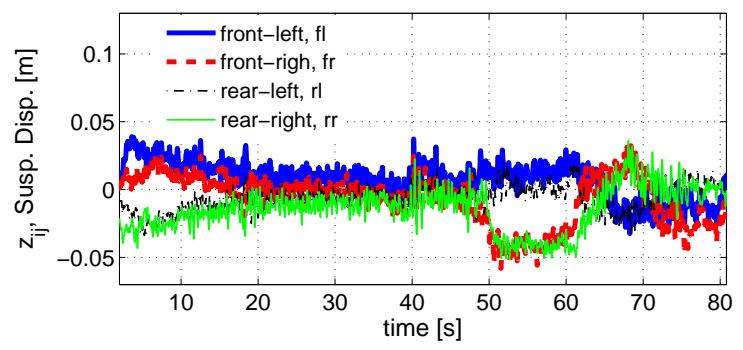

Fig. 9: Suspension height measurements on the banked road

The suspension height displacement measurements depicted in Fig. 9 have large fluctuations, but the suggested vehicle angle estimators (6) reject outliers and provide smooth vehicle roll and pitch angles as demonstrated in Fig. 10-a. Finally, the proposed estimator detects the road bank around the region of $t=50[s]$ as illustrated in Fig. 10-b.

The estimation results shown in Fig. 10-b confirm that even with the presence of the vehicle body angles around $t=5$ and $t=20[s]$, the developed estimator can successfully differentiate between the road and the body angles generated by lateral excitations and does not provide any road bank. The observed deviations around $t=65[\mathrm{~s}]$ may be contributed to improper selection of $E_{q}, F_{q}$ matrices and estimated vehicle velocities.

Case 3: Steering on a combined grade/bank

The performance of the unknown input observer on the roads with combined bank and grade angles is investigated in this case study. The maneuver includes driving on a graded road, lateral excitations by steering, and steering on a road with combined bank and grade. Estimating the road angles in this maneuver is challenging since the lateral excitations by the driver is performed on the combined banked/graded road. Figure 11 illustrates lateral and longitudinal excitations of the vehicle between $t=35$ and $t=92[s]$, which includes both the driver and road excitations.

Measured suspension height displacements are provided in 

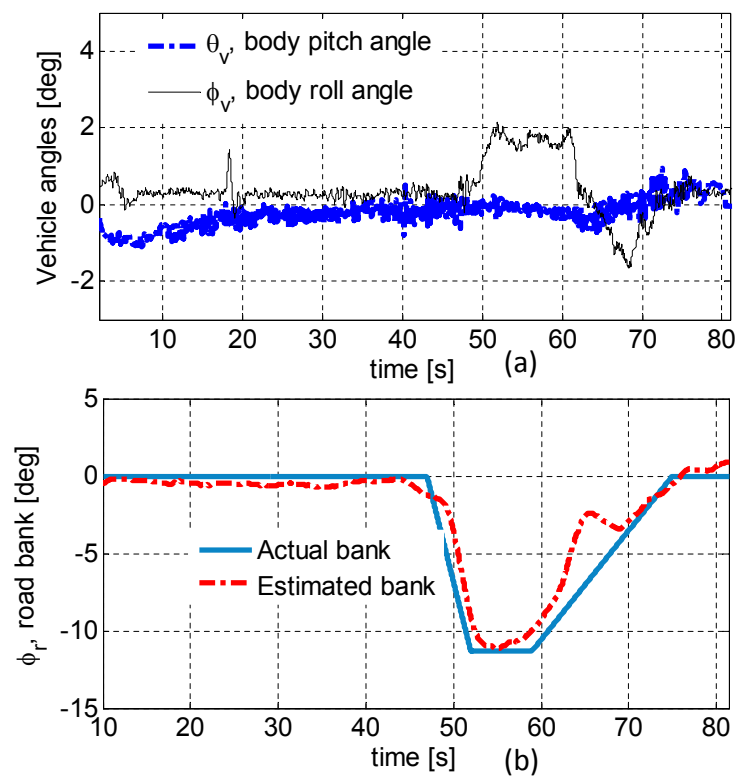

Fig. 10: Estimation results for Case2: (a) vehicle angles and (b) bank angle

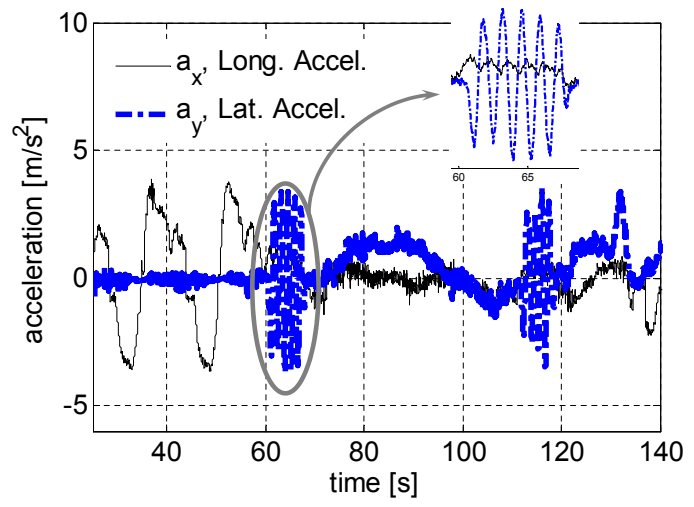

Fig. 11: Acceleration measurement on combined grade/bank

Fig. 12, which depicts lateral and longitudinal excitations. Suspension height displacements are smooth and no outlier is detected.

Successive excitations between $t=60$ and $t=70[\mathrm{~s}]$ are because of the sine steering on the graded road. The results of the calculated vehicle angles $\bar{\phi}_{v}, \bar{\theta}_{v}$ are shown in Fig. 13-a.

Despite the fact that the lateral excitations happened on the road with inclinations, the proposed unknown input observer can detect the road angles and distinguish between the body and road angles as depicted in Fig. 13-b. In spite of several pitch excitations (acceleration and brake) for $t \leq 60[s]$, the proposed UIO distinguishes between the road grade and vehicle body pitch angles and detects the road grade. Thus, the body pitch/roll does not affect this method significantly and is implemented as inputs in (20) and (21) to identify the road angles.

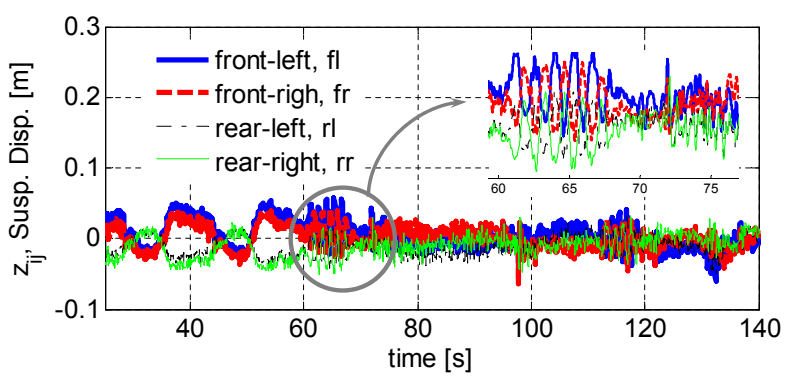

Fig. 12: Suspension height measurements on combined grade/bank
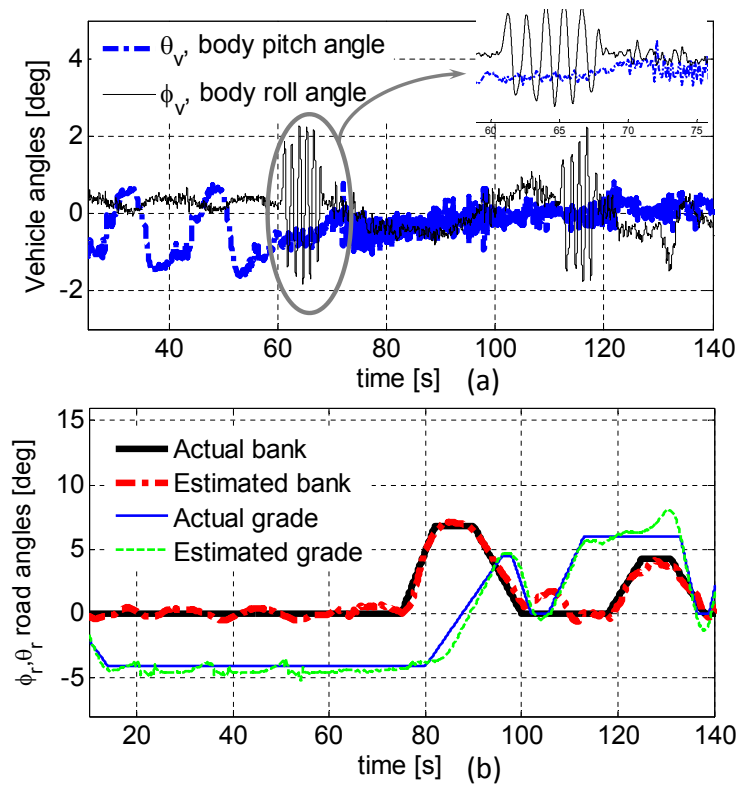

Fig. 13: Road experiments: (a) estimated vehicle angles on combined grade/bank and (b) estimated road angles

The experiments on various roads and with different drivers' inputs demonstrate that the proposed UIO can reject the outliers due to uneven road conditions at each corner and estimate the road angles.

\section{CONCLUSION}

This paper sets out to estimate the road angles and has proposed and experimentally tested a real-time structure for the estimation of road bank and grade angles. The suggested algorithm includes an unknown input observer on the roll and pitch dynamics of the vehicle. Observer gain matrices are designed to guarantee a fast convergence rate and satisfy (16) and (17). Road disturbances and outliers are isolated in the provided method using a dynamic threshold based on the longitudinal and lateral excitations of the vehicle. Incorporating road-body kinematics helped to achieve more accurate vehicle angle measurements. Road angle estimation 
in maneuvers with high excitation on banked/graded roads, fast convergence and robustness against harsh excitations, road disturbances, and outliers are among the advantages of the proposed methodology. Based on the developed unknown input observer and the performance of the road experiments on an instrumented vehicle, the following conclusions can be made:

The proposed algorithm can estimate different types of separate and combined bank/grad road angles in various driving conditions.

The observer does not require any information about the road friction, tire forces, and tire parameters. This enables the algorithm to perform reliably on different road conditions.

The current state estimators on the instrumented test vehicle (platform) are robust to errors in the road angle estimation up to $2[\mathrm{deg}]$ and the developed UIO exhibits errors less than this value. However, there is a $4.1[\mathrm{deg}]$ deviation around $t=65[s]$ in Case2 (Fig. 10) which may be due to the errors in estimated vehicle velocities and inappropriate selection of the UIO gain matrices that will be addressed in future. The proposed algorithm can be integrated with various active safety systems (e.g. stability control and rollover prevention systems), and vehicle state estimators to ensure the reliable performance of such systems in the presence of accelerations, roll rate, and pitch rate measurements affected by road inclinations.

\section{APPENDIX}

Observability and invertibility matrices: the following includes the invertibility and observability matrices used in Section II. The discrete-time output equation (11) can be rewritten as follows by iterating over $L+1$ time steps:

$$
\begin{aligned}
& {\left[\begin{array}{c}
y_{q}[0] \\
y_{q}[1] \\
y_{q}[2] \\
\vdots \\
y_{q}[L]
\end{array}\right]=\left[\begin{array}{c}
\bar{C}_{q} \\
\bar{C}_{q} \bar{A}_{q} \\
\bar{C}_{q} \bar{A}_{q}^{2} \\
\vdots \\
\bar{C}_{q} \bar{A}_{q}^{L}
\end{array}\right] x[0]+} \\
& {\left[\begin{array}{ccccc}
\bar{D}_{q} & 0 & 0 & \cdots & 0 \\
\bar{C}_{q} \bar{B}_{q} & \bar{D}_{q} & 0 & \cdots & 0 \\
\bar{C}_{q} \bar{A}_{q} \bar{B}_{q} & \operatorname{bar} C_{q} \bar{B}_{q} & \bar{D}_{q} & \cdots & 0 \\
\vdots & \vdots & \vdots & \ddots & \vdots \\
\bar{C}_{q} \bar{A}_{q}^{L-1} \bar{B}_{q} & \operatorname{barC}_{q} \bar{A}_{q}^{L-2} \bar{B}_{q} & \bar{C}_{q} \bar{A}_{q}^{L-3} \bar{B}_{q} & \bar{D}_{q}
\end{array}\right]\left[\begin{array}{c}
u_{q}[0] \\
u_{q}[1] \\
u_{q}[2] \\
\vdots \\
u_{q}[L]
\end{array}\right]}
\end{aligned}
$$

Therefore, the output equation (A1) can be expressed as the observability and invertibility matrices:

$$
y_{q}[0: L]=\mathcal{O}_{L q} x[0]+\mathcal{J}_{L q} u_{q}[0: L]
$$

Unknown input observer gain matrices: the implemented UIO gain matrices for the road experiments are obtained by stable pole placement in $E_{q}=\bar{A}_{q}-\bar{B}_{q} \breve{W}_{q}-\bar{M}_{q} \bar{W}_{q}$.
Implementing the pitch dynamics yields to:

$$
\begin{array}{r}
E_{\theta}=\left[\begin{array}{cc}
3.330 e-4 & 1.301 e-6 \\
-1.734 e-6 & 0.026
\end{array}\right], \\
F_{\theta}=\left[\begin{array}{cccc}
6.33 e-4 & 1.572 e-6 & 0.999 & 1.592 e-6 \\
6.458 e-3 & -0.0259 & -6.458 e-3 & 1.0
\end{array}\right] .
\end{array}
$$

The roll dynamics also results into the following UIO gain matrices:

$$
\begin{array}{r}
E_{\phi}=\left[\begin{array}{ccc}
3.340 e-4 & 1.297 e-6 \\
1.746 e-6 & 0.0258
\end{array}\right], \\
F_{\phi}=\left[\begin{array}{cccc}
6.294 e-4 & 1.558 e-6 & 0.997 & 1.579 e-6 \\
6.487 e-3 & -0.0259 & -6.487 e-3 & 1.0
\end{array}\right],
\end{array}
$$

\section{ACKNOWLEDGEMENT}

This work was supported by Automotive Partnership Canada, Ontario Research Fund, and the General Motors Co. [grant numbers APCPJ 39599609 and ORF-RE-04-039].

\section{REFERENCES}

[1] L. Imsland, T. A. Johansen, T. I. Fossen, H. F. Grip, J. C. Kalkkuhl, and A. Suissa, "Vehicle velocity estimation using nonlinear observers," Automatica, vol. 42, no. 12, pp. 2091-2103, 2006.

[2] L.-Y. Hsu and T.-L. Chen, "Vehicle full-state estimation and prediction system using state observers," Vehicular Technology, IEEE Transactions on, vol. 58, no. 6, pp. 2651-2662, 2009.

[3] S. Antonov, A. Fehn, and A. Kugi, "Unscented kalman filter for vehicle state estimation," Vehicle System Dynamics, vol. 49, no. 9, pp. 14971520, 2011.

[4] H. E. Tseng, "Dynamic estimation of road bank angle," Vehicle System Dynamics, vol. 36, no. 4-5, pp. 307-328, 2001.

[5] L.-H. Zhao, Z.-Y. Liu, and H. Chen, "Sliding mode observer for vehicle velocity estimation with road grade and bank angles adaptation," in Intelligent Vehicles Symposium, 2009 IEEE. IEEE, 2009, pp. 701706.

[6] L. Menhour, D. Lechner, and A. Charara, "Design and experimental validation of linear and nonlinear vehicle steering control strategies," Vehicle System Dynamics, vol. 50, no. 6, pp. 903-938, 2012.

[7] H. Faer Grip, L. Imsland, T. A. Johansen, J. C. Kalkkuhl, and A. Suissa, "Vehicle sideslip estimation: Design, implementation, and experimental validation," IEEE control systems, vol. 29, no. 5, pp. 36-52, 2009.

[8] H. F. Grip, L. Imsland, T. Johansen, J. C. Kalkkuhl, A. Suissa et al., "Estimation of road inclination and bank angle in automotive vehicles," in American Control Conference, 2009. ACC'09. IEEE, 2009, pp. 426-432.

[9] J.-o. Hahn, R. Rajamani, S. You, and K. Lee, "Road bank angle estimation using disturbance observer," in Proceedings of AVEC 6th International Symposium, 2002.

[10] L. Imsland, H. F. Grip, T. A. Johansen, T. I. Fossen, J. C. Kalkkuhl, and A. Suissa, "Nonlinear observer for vehicle velocity with friction and road bank angle adaptation-validation and comparison with an extended kalman filter," SAE Technical Paper, Tech. Rep., 2007.

[11] L. Imsland, T. A. Johansen, H. F. Grip, and T. I. Fossen, "On non-linear unknown input observers-applied to lateral vehicle velocity estimation on banked roads," International Journal of Control, vol. 80, no. 11, pp. 1741-1750, 2007. 
[12] J. Kim, H. Lee, and S. Choi, "A robust road bank angle estimation based on a proportional-integral h filter," Proceedings of the Institution of Mechanical Engineers, Part D: Journal of automobile engineering, p. 0954407011430919, 2012.

[13] C. R. Carlson and J. C. Gerdes, "Optimal rollover prevention with steer by wire and differential braking," in ASME 2003 International Mechanical Engineering Congress and Exposition. American Society of Mechanical Engineers, 2003, pp. 345-354.

[14] J. Ryu, E. J. Rossetter, and J. C. Gerdes, "Vehicle sideslip and roll parameter estimation using gps," in Proceedings of the AVEC International Symposium on Advanced Vehicle Control, 2002.

[15] L.-Y. Hsu and T.-L. Chen, "Estimating road angles with the knowledge of the vehicle yaw angle," Journal of dynamic systems, measurement, and control, vol. 132, no. 3, p. 031004, 2010.

[16] ISO, Road vehicles - Vehicle dynamics and road-holding ability Vocabulary, ISO 8855:2011, 2011

[17] M. Darouach, M. Zasadzinski, and S. J. Xu, "Full-order observers for linear systems with unknown inputs," IEEE transactions on automatic control, vol. 39, no. 3, pp. 606-609, 1994.

[18] M. E. Valcher, "State observers for discrete-time linear systems with unknown inputs," Automatic Control, IEEE Transactions on, vol. 44 no. 2, pp. 397-401, 1999.

[19] S. Sundaram and C. N. Hadjicostis, "Delayed observers for linear systems with unknown inputs," Automatic Control, IEEE Transactions on, vol. 52, no. 2, pp. 334-339, 2007.

[20] A. Cristofaro and T. A. Johansen, "Fault tolerant control allocation using unknown input observers," Automatica, vol. 50, no. 7, pp. 1891-1897, 2014.

[21] C. Robinson and P. Kumar, "Sending the most recent observation is not optimal in networked control: Linear temporal coding and towards the design of a control specific transport protocol," in Decision and Control, 2007 46th IEEE Conference on. IEEE, 2007, pp. 334-339.

[22] S. Sundaram and C. N. Hadjicostis, "Partial state observers for linear systems with unknown inputs," Automatica, vol. 44, no. 12, pp. 3126 3132, 2008.

[23] S. Ahmadizadeh, J. Zarei, and H. R. Karimi, "Robust unknown input observer design for linear uncertain time delay systems with application to fault detection," Asian Journal of Control, vol. 16, no. 4, pp. 1006 1019,2014

[24] E. Hashemi, M. Pirani, A. Khajepour, B. Fidan, A. Kasaiezadeh, S. Chen, and B. Litkouhi, "Integrated estimation structure for the tire friction forces in ground vehicles," in Advanced Intelligent Mechatronics (AIM), 2016 IEEE International Conference on. IEEE, 2016, pp. 1657-1662.

[25] G. Baffet, A. Charara, and G. Dherbomez, "An observer of tire-road forces and friction for active security vehicle systems," Mechatronics, IEEE/ASME Transactions on, vol. 12, no. 6, pp. 651-661, 2007.

[26] F. Sun, K. Lolenko, and J. Rudolph, "Nonlinear observer design for state estimation during antilock braking," Proceedings of the Institution of Mechanical Engineers, Part I: Journal of Systems and Control Engineering, vol. 228, no. 2, pp. 78-86, 2014.

[27] M. Gadola, D. Chindamo, M. Romano, and F. Padula, "Development and validation of a kalman filter-based model for vehicle slip angle estimation," Vehicle System Dynamics, vol. 52, no. 1, pp. 68-84, 2014.

[28] E. Hashemi, A. Kasaiezadeh, S. Khosravani, A. Khajepour, N. Moshchuk, and S.-K. Chen, "Estimation of longitudinal speed robust to road conditions for ground vehicles," Vehicle System Dynamics, vol. 54, no. 8, pp. 1120-1146, 2016.

[29] G. F. Franklin, J. D. Powell, and M. L. Workman, Digital Control of Dynamic Systems. Menlo Park: Addison Wesley Longman, 1998.

[30] A. S. Willsky, "On the invertibility of linear systems," IEEE Transactions on Automatic Control, vol. 19, no. 3, pp. 272-274, 1974. 